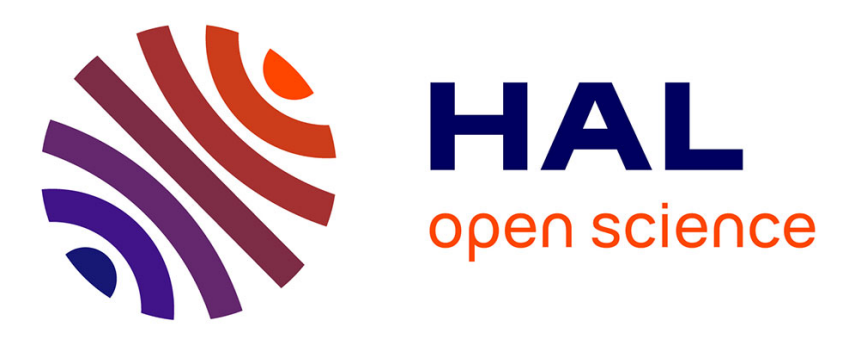

\title{
Three-dimensional turbulence generated homogeneously by magnetic particles
}

\author{
Annette Cazaubiel, Jean-Baptiste Gorce, Jean-Claude Bacri, Michaël \\ Berhanu, Claude Laroche, Eric Falcon
}

\section{- To cite this version:}

Annette Cazaubiel, Jean-Baptiste Gorce, Jean-Claude Bacri, Michaël Berhanu, Claude Laroche, et al.. Three-dimensional turbulence generated homogeneously by magnetic particles. Physical Review Fluids, 2021, 6, pp.L112601. 10.1103/PhysRevFluids.6.L112601 . hal-03400228

\section{HAL Id: hal-03400228 \\ https://hal.science/hal-03400228}

Submitted on 24 Oct 2021

HAL is a multi-disciplinary open access archive for the deposit and dissemination of scientific research documents, whether they are published or not. The documents may come from teaching and research institutions in France or abroad, or from public or private research centers.
L'archive ouverte pluridisciplinaire HAL, est destinée au dépôt et à la diffusion de documents scientifiques de niveau recherche, publiés ou non, émanant des établissements d'enseignement et de recherche français ou étrangers, des laboratoires publics ou privés. 


\title{
Three-dimensional turbulence generated homogeneously by magnetic particles
}

\author{
A. Cazaubiel, J.-B. Gorce, J.-C. Bacri, M. Berhanu, C. Laroche, and E. Falcon? \\ Université de Paris, MSC, UMR 7057 CNRS, F-75 013 Paris, France
}

\begin{abstract}
Three-dimensional turbulence is usually studied experimentally by using a spatially localized forcing at large scales (e.g. via rotating blades or oscillating grids), often in a deterministic way. Here, we report an original technique where the fluid is forced in volume, randomly in space and time, using small magnetic particles remotely driven. Such a forcing generates almost no mean flow and is closer to those of direct numerical simulations of isotropic homogeneous turbulence. We compute the energy spectra and structure functions using local and spatiotemporal flow velocity measurements. The energy dissipation rate is also evaluated consistently in five different ways. Our experimental results confirm the stationary, homogeneous and isotropic features of such turbulence, and in particular the Tennekes' model for which the Tennekes' constant is experimentally estimated.
\end{abstract}

\section{INTRODUCTION}

Turbulence concerns swirling motions of fluids occurring irregularly in space and time. This phenomenon occurs in most geophysical or astrophysical flows, as well as in many industrial processes [1, 2]. However, attempts to find analytical solutions to the forced Navier-Stokes equations in a turbulent regime still remain unsuccessful. Three-dimensional turbulence is thus mainly described phenomenologically using dimensional and similarity arguments assuming notably homogeneity, isotropy and statistical stationarity [1, 3 $[$ 6]. For a long time, 3D turbulence experiments consisted of uniform grids of bars in a wind tunnel (freely decaying turbulence) to get closer to ideal isotropic and homogeneous turbulence [1, 5]. Nowadays, most laboratory experiments on 3D stationary turbulence are performed in a closed container where energy is injected from a boundary of the container, at large scales and often in a deterministic way, such as oscillating grids [7-10], counter-rotating disks (von Kármán flow) [11], several fans [12] or propellers [13], or multiple jets 14-16]. In contrast, direct numerical simulations of 3D turbulence use a forcing in volume either in spectral space [17, or more recently in physical space [18. To be able to experimentally force turbulence in the whole volume of a container (if possible randomly in time and space) is a challenge that has never been achieved to our knowledge, and would thus lead to a better comparison with direct numerical simulations [19].

Here, we present an original forcing technique where the fluid is forced in volume randomly in space and time, by using small magnetic particles remotely driven. An external oscillating magnetic field drives stochastic rotation of each magnetic particle, whereas the collisions between particles or with the container boundaries lead to erratic translational motions. Such a forcing within the bulk favors the statistical homogeneity of the velocity field with nearly no mean flow. The measured energy spectra, structure functions and energy dissipation rate (evaluated consistently in five different ways) confirm the stationary, homogeneous and isotropy features of such generated turbulence that could be easily implemented in different domains.

Beyond its implementation to measure global dissipated power in 3D turbulence [20], this forcing mechanism can be also easily used in other systems as in soft matter to study a 3D granular "gas" in air (showing several major differences with a boundary-driven system) 21]. Furthermore, colloidal magnetic spinners on a fluid surface, as well as active (self-propelled) swimmers, can generate flow reminiscent of 2D turbulence [22, 23].

\section{THEORETICAL BACKGROUNDS}

For large enough Reynolds numbers and 3D stationary, homogeneous, and isotropic turbulence, the energy spectrum is predicted dimensionally as $E(k)=\mathcal{C} \epsilon^{2 / 3} k^{-5 / 3}[3]$ with $\epsilon$ the energy dissipation rate per unit mass and $k$ the Fourier spatial scale, and $\mathcal{C} \approx 1.6$ the Kolmogorov constant measured experimentally [5, 24]. $\epsilon$ also represents the mean flux of kinetic energy cascading from the large (forcing) scale to the small (dissipative) scale. This energy transfer through this inertial range is due to nonlinearity. The unidimensional (transverse and longitudinal) energy spectra are proportional theoretically as $E_{\perp}\left(k_{x}\right)=4 / 3 E_{\|}\left(k_{x}\right)$ with $E_{\|}\left(k_{x}\right)=C \epsilon^{2 / 3} k_{x}^{-5 / 3}$ and $C=18 \mathcal{C} / 55[\underline{5}, 24]$. The second-order moment of the velocity increments at a distance $r$ (or structure function) $\mathcal{S}_{2}(r) \equiv\left\langle[v(x+r)-v(x)]^{2}\right\rangle$ is dimensionally predicted as $\mathcal{S}_{2}(r)=C_{2} \epsilon^{2 / 3} r^{2 / 3}$ [3], $x$ is a spatial coordinate, and $C_{2} \approx 2.0$ is an experimentally

* E-mail: eric.falcon@u-paris.fr 


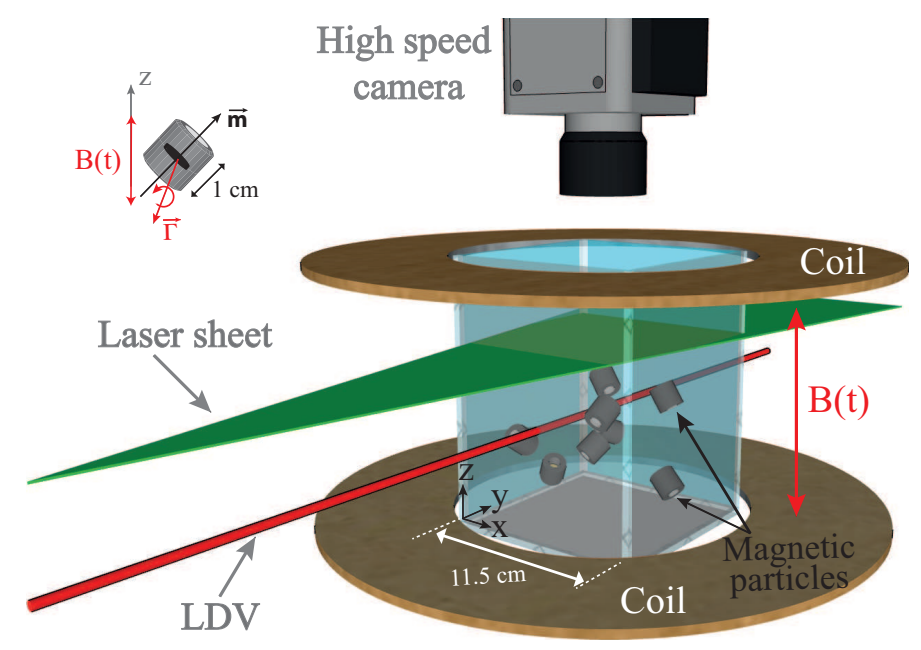

FIG. 1. Experimental setup showing the 3D container of fluid and the encapsulated magnets together with PIV and LDV measurements. Top left: enlargement of a magnetic particle. A vertical oscillating magnetic field $B(t)$ drives time-dependent rotations of each magnetic particle by applying a torque $\vec{\Gamma}$ over its magnetic moment $\overrightarrow{\mathrm{m}}$.

measured constant [5]. The third-order structure function is analytically derived as $\mathcal{S}_{3}(r)=-4 / 5 \epsilon r($ the only exact result known for turbulence) called Kolmogorov's 4/5 law [4]. Finally, intermittency occurs if the structure functions of order $p, \mathcal{S}_{p}(r) \equiv\left\langle[v(x+r)-v(x)]^{p}\right\rangle$, scales as $r \zeta_{p}$ with a nonlinear dependence of $\zeta_{p}$ with $p$ [25], instead of $\zeta_{p}=p / 3$ [3] . For finite Reynolds numbers, the previous laws have several corrections [], 26] .

\section{EXPERIMENTAL SETUP}

The experimental setup is shown in Fig. 1. A Plexiglas square-section container of length $L=11.5 \mathrm{~cm}$ and height $h=9 \mathrm{~cm}$, is filled with distilled water. $N$ home-made magnetic particles are put within the container $(N \in[1,60])$. Each particle is made of a cylindrical permanent neodymium magnet (NdFeB, N52, $0.5 \mathrm{~cm}$ in diameter, $0.2 \mathrm{~cm}$ in thickness) encased and axially aligned in a cylindrical Plexiglas shell (1 cm in outer diameter and $1 \mathrm{~cm}$ long) to strongly reduce dipolar interaction between particles [20]. The container is sealed with a transparent lid and sits between two Helmholtz coils powered by a sinusoidal current of amplitude $I \in[0,9]$ A and frequency $F \in[0,50]$ Hz. A vertical oscillating magnetic field $B(t)=B \sin (2 \pi F t)$ is thus generated with an amplitude $B \in[0,207] \mathrm{G}$ measured with a gaussmeter (FW Bell). $B$ is spatially homogeneous in the container volume with a $5 \%$ accuracy. The AC magnetic field transfers angular momentum into each particle which is converted into linear momentum during collisions, leading to erratic translational and rotational motions of the particles (see [20] for details). The fluid is thus forced homogeneously in volume, and randomly in both space and time. The fluid velocity is measured in a single point over time by nonintrusive Laser Doppler Velocimetry (LDV Dantec Flow Explorer 1D) to access to its frequency spectrum. The fluid velocity field is measured in a horizontal $x y$ plane $\left(11 \times 9 \mathrm{~cm}^{2}\right)$ over time by Particle Image Velocimetry (PIV) [27], in particular to access the wavenumber spectrum and structure functions. The fluid flow is visualized using Polyamide fluid tracers $(50 \mu \mathrm{m})$ illuminated by a horizontal laser sheet. A high-resolution video camera (Phantom V10, $2400 \times 1800$ pixel $^{2}$ at $200 \mathrm{fps}$ ), located on the top of the fluid container, records the motion of the fluid tracers. The spatial resolution is $0.8 \mathrm{~mm}$ (i.e., spacing between adjacent velocity field vectors). Note that less than $3 \%$ of the acquired images are discarded and correspond to rare events of a magnetic particle passing through the laser sheet. This leads to experiments for PIV with a lower $N$ and at lower fluid RMS velocity $\left(\sigma_{u} \leq 4 \mathrm{~cm} / \mathrm{s}\right)$ than for $\operatorname{LDV}\left(\sigma_{u} \leq 18 \mathrm{~cm} / \mathrm{s}\right)$. For most of the results presented below, the volume fraction is $0.7 \%$ (corresponding to $N=10)$.

Figure 2 shows the typical fluid motions characteristic of a turbulent flow (see also movies in the Supplemental Material [28]). Strong spatial and temporal fluctuations of the flow are observed over various scales, together with eddies. We will characterize hereafter the properties of such turbulent flow generated by this novel forcing. We will also verify if a self-similar energy transfer through the scales occurs by nonlinearity. 


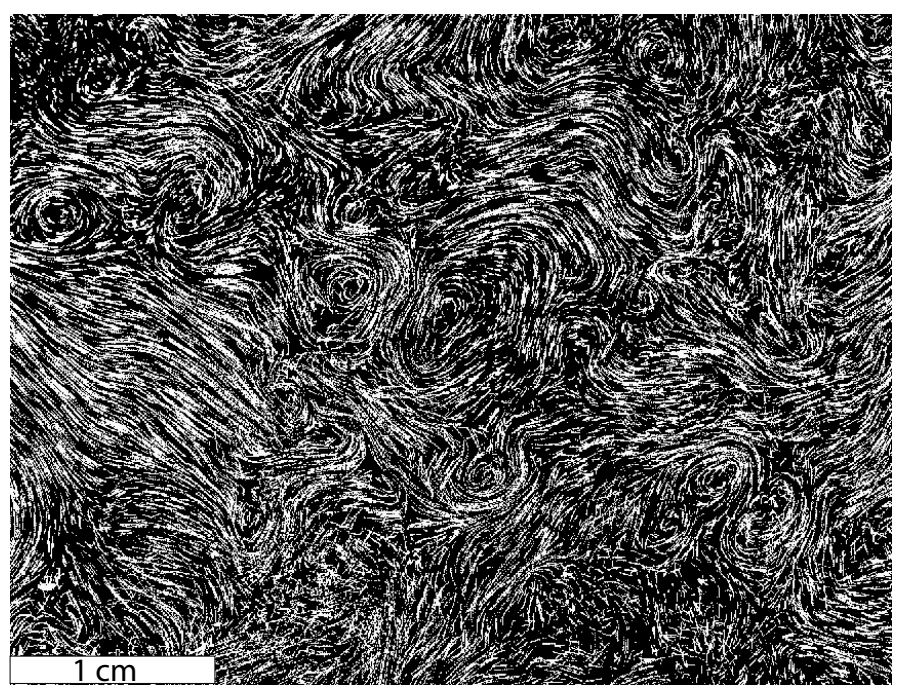

FIG. 2. Fluid tracer trajectories within the laser sheet followed over 10 consecutive images (0.05 s). Forcing parameters: $N=10, B=115 \mathrm{G}$, and $F=50 \mathrm{~Hz} . \sigma_{u}=2.3 \mathrm{~cm} / \mathrm{s}$.

\section{HOMOGENEITY, ISOTROPY AND LEVEL OF TURBULENCE WITH CONTROL PARAMETERS}

The longitudinal and transverse horizontal fluid velocities at a location $x$ are defined as $u(x, t)$ and $v(x, t)$, the vertical one is $w(x, t)$. Using PIV, we first check that the RMS fluctuating velocity $\sigma_{u}$ is well invariant by translation in the $x y$ plane, and by rotation of the latter, meaning thus that the velocity field is homogeneous and isotropic in the horizontal plane. The isotropy ratios are indeed $\sigma_{u} / \sigma_{v}=0.97 \pm 0.01$ and $\sigma_{u} / \sigma_{w}=0.87 \pm 0.01$. Moreover, the mean velocity $\langle u\rangle_{t, x}$ is found to be much smaller than the RMS fluctuations (i.e., $\langle u\rangle_{t, x} /\left\langle\sigma_{u}\right\rangle_{x}<11 \%$ ) to be able to neglect afterward the mean flow (see the Supplemental Material [28]).

Using single-point LDV measurements, we now focus on the scalings of the fluid velocity fluctuations with the forcing parameters (number of magnetic particles $N$, amplitude $B$ and frequency $F$ of the magnetic field). The fluid RMS velocity fluctuations $\sigma_{u}=\sqrt{\left\langle u^{2}\right\rangle_{t}}$ are found to depend on the forcing parameters as $\sigma_{u} \sim N^{1 / 2} B^{1 / 3} F^{1 / 3}$ (see the Supplemental Material [28]). The magnetic particle velocity was previously found to scale as $V_{p} \sim N^{0} B^{1 / 3} F^{1 / 3}$ from the power budget between the injected power into the fluid by the magnetic particles and the power dissipated [20]. The latter is mainly due to viscous dissipation by a turbulent translational drag on the particles and by inelastic collisions between particles (or with the container walls) [20]. Assuming that the kinetic energy of the fluid is proportional to the particle ones $\sim N V_{p}^{2}$, the RMS fluid velocity scales indeed as $\sigma_{u} \sim\left(N V_{p}^{2}\right)^{1 / 2} \sim N^{1 / 2} B^{1 / 3} F^{1 / 3}$.

\section{FREQUENCY SPECTRUM}

The power spectrum density $S_{u}(f)$ of the fluid velocity $u(t)$ measured by LDV is shown in Fig. 3 and compensated by $f^{-5 / 3}$ for an increasing number $N$ of magnetic particles at fixed $B$ and $F$. The spectrum amplitude increased with $N$. More importantly, each spectrum follows a frequency power-law in $f^{-5 / 3}$ over more than one decade in frequency. For zero-mean velocity flows, Tennekes' model (large-scale advection of turbulent eddies) predicts the frequency spectrum to scale as $f^{-5 / 3}[29]$, as observed here. More precisely, one would expect $S(\omega)=\beta \epsilon^{2 / 3} q^{2 / 3} \omega^{-5 / 3}$ with $\beta$ an empirical constant and $q \equiv \sqrt{\left(\sigma_{u}^{2}+\sigma_{v}^{2}+\sigma_{w}^{2}\right)}$ [29]. Since $\epsilon \sim \sigma_{u}^{3}$ (see below), $S(\omega)$ has to scale as $\sigma_{u}^{8 / 3}$. We thus plot in the inset of Fig. [3 the compensated spectra $S_{u}(f) f^{5 / 3}$ rescaled by $N^{4 / 3} B^{8 / 9} F^{8 / 9}$ for a large range of forcing parameters $(N, B$ and $F)$. All rescaled spectra are well superimposed on a master curve with a plateau over more than one decade. As we confirm the Tennekes' model, we are then able to infer experimentally the Tennekes' constant from the compensated spectra and $\epsilon$ values. One finds $\beta=0.64 \pm 0.15$. This value confirms the assumed Tennekes' constant of the order of 1 [29] and simulations leading to $\beta=0.82[30]$. Note that the rare previous experimental estimates (mainly on smaller inertial ranges) vary from $\beta=0.14$ [16] (resp. 0.23 [15]) using multiple jets forcing, without (resp. with) a free surface, to $\beta \in[0.48,0.62][8]$ and $5.5[9]$ using oscillating grids forcing but without PIV measurements, or $\beta \in[0.28,3.5]$ for low Reynolds number flows [10]. 


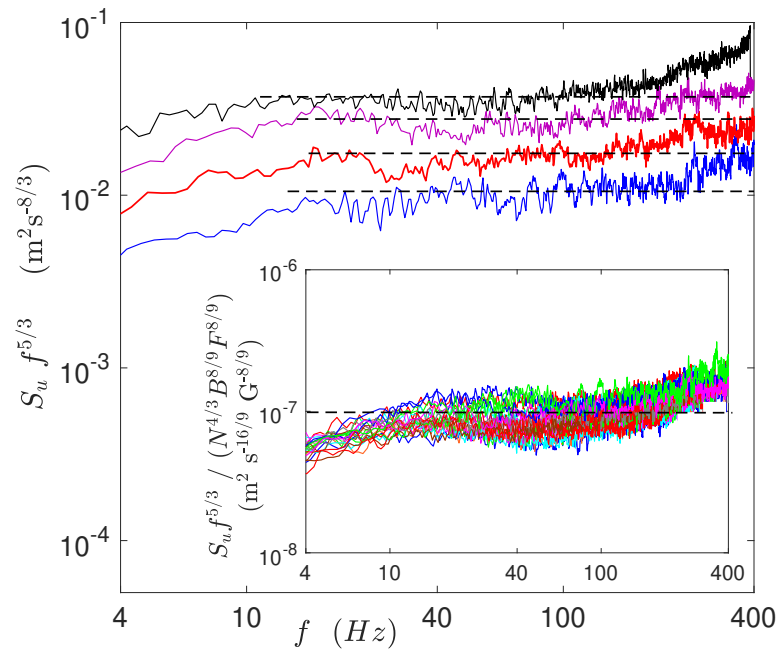

FIG. 3. Frequency power spectrum of the velocity $u(t)$ compensated by $f^{-5 / 3}, S_{u}(f) f^{5 / 3}$, for different $N$ from 10 (bottom) to 60 (top), with $F=30 \mathrm{~Hz}$ and $B=161 \mathrm{G}$. Dashed lines correspond to the predictions (see text). Inset: compensated power spectra, $S_{u}(f) f^{5 / 3}$ rescaled by $N^{4 / 3} B^{8 / 9} F^{8 / 9}$ for various $N \in[10,60], B \in[103,184] \mathrm{G}$, and $F \in[5,55] \mathrm{Hz}$.

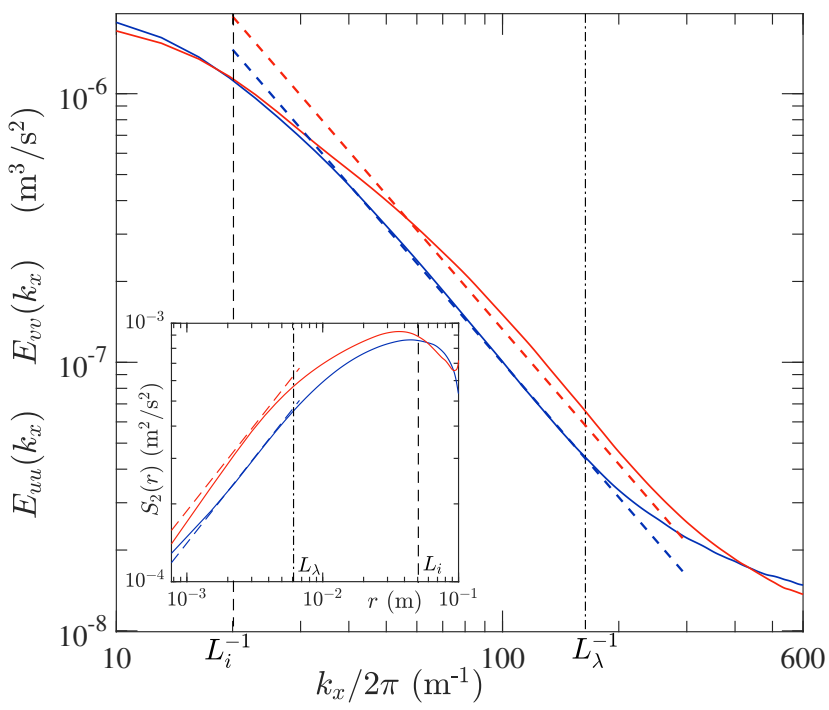

FIG. 4. 1D wavenumber power spectrum $E_{u u}\left(k_{x}\right)$ (in blue) and $E_{v v}\left(k_{x}\right)$ (red) in the $x$ direction of the velocity components $u$ and $v . N=10, B=115 \mathrm{G}$ and $F=50 \mathrm{~Hz} . \sigma_{u}=2 \mathrm{~cm} / \mathrm{s}$. Dashed lines: $k_{x}^{-5 / 3}$ prediction (blue one is adjusted and red one is inferred from the prediction $E_{v v}=\frac{4}{3} E_{u u}$ ). Vertical lines correspond to the length scales $L_{i}$ (dotted line) and $L_{\lambda}$ (dotted-dash line). Inset: Second-order structure functions $\mathcal{S}_{2}^{(u)}(r)$ (blue) and $\mathcal{S}_{2}^{(v)}(r)$ (red). Blue dashed line: best fit of $\mathcal{S}_{2}^{(u)}$ in $r^{2 / 3}$. The red dashed line is derived from the blue line using the relationship $\mathcal{S}_{2}^{(v)}=\frac{4}{3} \mathcal{S}_{2}^{(u)}$.

\section{WAVENUMBER SPECTRUM AND CHARACTERISTIC SCALES}

Using PIV, the 1D wavenumber power spectra (in the $x$ direction), $E_{u u}\left(k_{x}\right)$ and $E_{v v}\left(k_{x}\right)$, of the longitudinal and transverse components $\left(u\right.$ and $v$ ) of the velocity field are shown in Fig. 4 . The longitudinal spectrum $E_{u u}\left(k_{x}\right)$ scales as $k_{x}^{-5 / 3}$ over a decade as expected from Kolmogorov's law $E_{u u}=C \epsilon^{2 / 3} k_{x}^{-5 / 3}[3]$. We also observe that the transverse spectrum $E_{v v}\left(k_{x}\right)$ is proportional to the longitudinal one in agreement with $E_{v v}\left(k_{x}\right)=\frac{4}{3} E_{u u}\left(k_{x}\right)$ [5] (see dashed lines in Fig. (4). The degree of isotropy is thus comparable with that in DNS where the same equivalence between 1D spectra is found [31].

The inertial scales of turbulence are located between the container size $L$ and the small dissipative Kolmogorov 


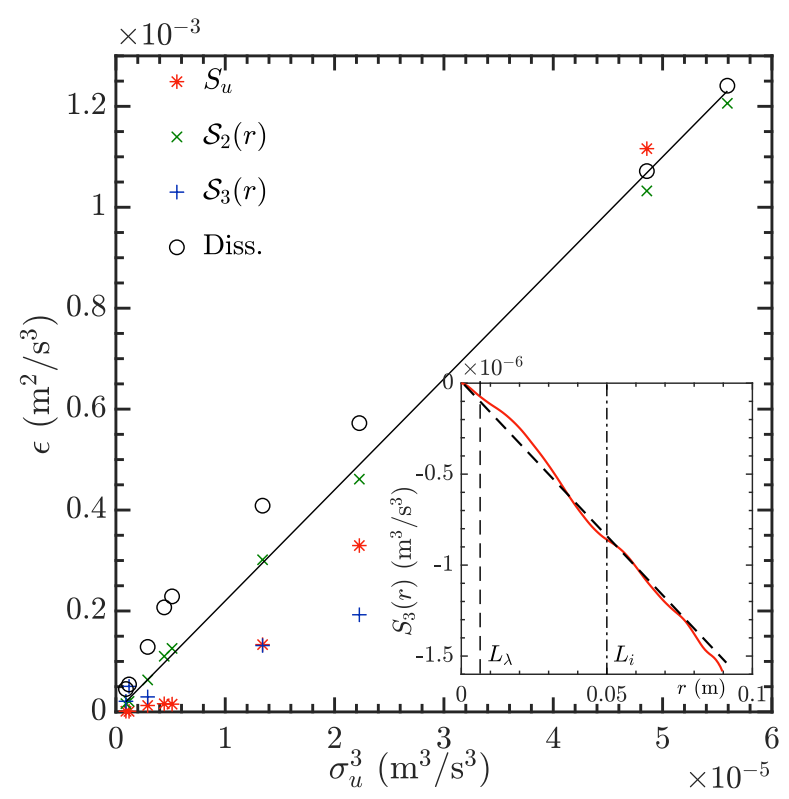

FIG. 5. Different estimations of $\epsilon$ as a function of $\sigma_{u}^{3}$ inferred from $(*)$ the wavenumber spectrum $E_{u u},(\times)$ the second-order structure function $\mathcal{S}_{2}(r),(+)$ the third-order structure function $\mathcal{S}_{3}(r)$, and (o) the dissipation rate definition (see text). Solid line is the prediction $c \sigma_{u}^{3} / L_{i}$ with $c=1.1$. Inset: Third-order structure function $\mathcal{S}_{3}(r)$ (solid line). Dashed line is a linear fit.

scale $\eta=\left(\nu^{3} / \epsilon\right)^{1 / 4}\left[\underline{3}\right.$. Here, one has $\eta \approx 0.2 \mathrm{~mm}$ for a typical mean dissipation rate $\epsilon=10^{-3} \mathrm{~m}^{2} \mathrm{~s}^{-3}$ (see below), $\nu=10^{-6} \mathrm{~m}^{2} \mathrm{~s}^{-1}$ is the fluid kinematic viscosity. The integral length scale can not be accurately computed from the autocorrelation function of the velocity field since the container size $L$ is not eight times larger than the integral scale [5, 33]. We evaluate the integral scale $L_{i}=5 \mathrm{~cm}$ from the abscissa of the maximum of $\mathcal{S}_{2}(r)$ (see inset of Fig. 44),

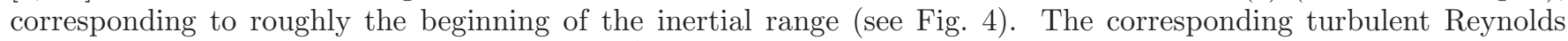
number at $L_{i}$ thus reads $R e_{L_{i}}=\sigma_{u} L_{i} / \nu \approx 10^{3}$, with $\sigma_{u}=2 \mathrm{~cm} / \mathrm{s}$. The Taylor length scale is estimated as $L_{\lambda} \approx 6$ mm (well located between $L_{i}$ and $\eta$ - see Fig. (4) using $L_{\lambda}=L_{i} \sqrt{15 / R e_{L_{i}}}$ [1]. The corresponding Taylor Reynolds number is $\operatorname{Re}_{\lambda}=\sigma_{u} L_{\lambda} / \nu \approx 122$, a value of the same order of magnitude as the ones in boundary forced turbulence experiments $[1] 14]$.

\section{STRUCTURE FUNCTIONS}

The structure functions of the velocity field are also computed from the PIV measurements. The inset of Fig. 4 shows the second-order structure functions $\mathcal{S}_{2}(r)$ in the $x$ direction of the horizontal components of the velocity field $u$ and $v$. The structure functions $\mathcal{S}_{2}(r)$ are roughly proportional to $r^{2 / 3}$ in the inertial range, as expected by the $2 / 3$ Kolmogorov's law (see above) [3]. Moreover, as for the spectra, the transverse and longitudinal components are found proportional as $\mathcal{S}_{2}^{(v)}=\frac{4}{3} \mathcal{S}_{2}^{(u)}$ (see dashed lines) as expected theoretically. From $\mathcal{S}_{2}^{(u)}$ and $E_{u u}$, one can also infer the ratio of the $2 / 3$ law constant over the Kolmogorov's constant, $C_{2} / C=5.3 \pm 2.8$, not so far from previous experimental evaluations $\approx 4[5]$. The third-order structure function $\mathcal{S}_{3}^{(u)}$ of the longitudinal velocity field is also computed and shown in the inset of Fig. $5 \mathcal{S}_{3}^{(u)}$ is found to decrease linearly with $r$ over one decade in the inertial range, in good agreement with the $4 / 5$ Kolmogorov's law [4] and with DNS [32]. This corresponds to the negative asymmetry of the velocity fluctuation gradients quantified by the skewness $\mathcal{S}_{3}^{(u)} /\left(\mathcal{S}_{2}^{(u)}\right)^{3 / 2}=-0.3 \pm 0.2$ close to the value inferred from the $4 / 5$ law, $-4 /\left(5 C_{2}\right)=-0.4[5]$.

\section{ENERGY DISSIPATION RATE}

Finally, the mean energy dissipation rate $\epsilon$ is estimated in five different ways: (i) as $E_{u u}^{3 / 2} k_{x}^{5 / 2} / C^{3 / 2}$ from the experimental 1D wavenumber spectrum and the Kolmogorov's spectrum, (ii) as $\left[\mathcal{S}_{2}^{(u)} / C_{2}\right]^{3 / 2} / r$ from the experimental $\mathcal{S}_{2}^{(u)}$ and the $2 / 3$ law, (iii) as $-5 \mathcal{S}_{3}^{(u)} /(4 r)$ from the experimental $\mathcal{S}_{3}^{(u)}$ and the $4 / 5$ law, (iv) from its definition for 
isotropic turbulence $\epsilon \equiv 15 \nu\left\langle\left(\partial u_{x} / \partial x\right)^{2}\right\rangle$ [5, 34], and (v) from dimensional analysis. These different estimations of $\epsilon$ are plotted in Fig. 5 as a function of $\sigma_{u}^{3}$. All $\epsilon$ values are found of the same order of magnitude at fixed $\sigma_{u}$, and are proportional to $\sigma_{u}^{3}$ regardless of the method used. Dimensional arguments estimate the dissipation rate from the velocity fluctuations as $\epsilon=c \sigma_{u}^{3} / L_{i}[34,35]$ involving the integral scale $L_{i}$, and $c$ a constant of the order of unity [36, 37]. Here, one finds $c=1.1$ close to the values found with a boundary forcing (grid turbulence) [35, 38]. These estimations of $\epsilon$ by five different methods are hardly obtained experimentally [39] and are found here to be all consistent as a consequence of the stationary, homogeneous and isotropic turbulence generated by this forcing in volume. Note that higher turbulence levels can be explored with this forcing (e.g., $\epsilon \sim 610^{-3} \mathrm{~m}^{2} / \mathrm{s}^{-3}$ for $\sigma_{u} \sim 0.18$ $\mathrm{m} / \mathrm{s}$ measured with LDV).

\section{CONCLUSION}

We developed an original technique to generate 3D turbulence by injecting energy in volume, randomly in time and space, by using small magnetic particles remotely driven. This forcing contrasts with previous ones in which a spatially localized forcing is applied at large-scale from a container boundary. We characterize the turbulence generated by this forcing in volume by local and spatiotemporal measurements of the fluid velocity. Almost no mean flow is involved, and all measured properties confirm the stationary, homogeneous and isotropic features of such turbulence. In particular, we confirm experimentally the Tennekes' model and resolve the disagreement between previously suggested value of the Tennekes' constant. Possible intermittency of such generated turbulence could be explored in the future [40], as well as its Lagrangian properties [41]. Moreover, this forcing mechanism is closer to those of direct numerical simulations and is rather flexible (e.g. either random in space and time or random only in space or only in time). It appears very promising to study large-scale 3D turbulence (i.e. larger than the injection scale) and its possible description by statistical mechanics tools [42]. It could be also applied to smart control of turbulence [43. Finally, this homogeneous forcing could be used to better explore geophysical- or astrophysical-like turbulent flows (rotating, stratified, or multiphase flows), and could provide a technological breakthrough in turbulent mixing.

\section{ACKNOWLEDGMENTS}

This work was supported by the French National Research Agency (ANR DYSTURB project No. ANR-17-CE300004), and by the Simons Foundation MPS N 651463 -Wave Turbulence.

[1] P. A. Davidson, Turbulence, 2nd Ed. (Oxford University Press, 2015).

[2] S. Galtier, Introduction to Modern Magnetohydrodynamics (Cambridge University Press, 2016).

[3] A. N. Kolmogorov, The local structure of turbulence in incompressible viscous fluid for very large reynolds numbers, Dokl. Akad. Nauk SSSR 30, 301 (1941), english translation in Proc. R. Soc. Lond. A 434, 9 (1991).

[4] A. N. Kolmogorov, Dissipation of energy in the locally isotropic turbulence, Dokl. Akad. Nauk SSSR 32, 16 (1941), english translation in Proc. R. Soc. Lond. A 434, 15 (1991).

[5] S. B. Pope, Turbulent Flows (Cambridge University Press, 2000).

[6] U. Frisch, Turbulence: The Legacy of A. N. Kolmogorov (Cambridge University Press, 1995).

[7] A. Srdic, H. J. S. Fernando, and L. Montenegro, Generation of nearly isotropic turbulence using two oscillating grids, Exp. Fluids 20, 395 (1996); E. Villermaux, B. Sixou, and Y. Gagne, Intense vortical structures in grid-generated turbulence, Phys. Fluids 7, 2008 (1995).

[8] E. Kit, H. J. S. Fernando, and J. A. Brown, Experimental examination of Eulerian frequency spectra in zero-mean-shear turbulence, Phys. Fluids 7, 5 (1995).

[9] I. P. D. De Silva, and H. J. S. Fernando, Oscillating grids as a source of nearly isotropic turbulence, Phys. Fluids 6, 2455 (1994).

[10] A. Al-Homoud, and M. Honzdo, Energy dissipation estimates in oscillating grid setup: LDV and PIV measurements, Environ. Fluid Mech. 7, 143 (2007).

[11] S. Douady, Y. Couder, and M. E. Brachet, Direct observation of the intermittency of intense vorticity filaments in turbulence, Phys. Rev. Lett. 67, 983 (1991); S. Fauve, C. Laroche, and B. Castaing, Pressure fluctuations in swirling turbulent flows, J. Phys. II France 3, 271 (1993); B. Saint-Michel, B. Dubrulle, L. Marié, F. Ravelet, and F. Daviaud, Evidence for Forcing-Dependent Steady States in a Turbulent Swirling Flow, Phys. Rev. Lett. 111, 234502 (2013).

[12] M. Birouk, B. Sarh, and I. Gökalp, An attempt to realize experimental isotropic turbulence at low reynolds number, Flow Turbulence Combust 70, 325 (2003). 
[13] M. Guala, A. Liberzon, K. Hoyer, A. Tsinober, and W. Kinzelbach, Experimental study on clustering of large particles in homogeneous turbulent flow, J. Turb. 9, N34 (2008); R. Zimmermann, H. Xu, Y. Gasteuil, M. Bourgoin, R. Volk, J.-F. Pinton, and E. Bodenschatz, The Lagrangian exploration module: An apparatus for the study of statistically homogeneous and isotropic turbulence, Rev. Sci. Instr. 81, 055112 (2010).

[14] W. Hwang and J. K. Eaton, Creating homogeneous and isotropic turbulence without a mean flow, Exp. Fluids 36, 444 (2004); K. Chang, G. P. Bewley, and E. Bodenschatz, Experimental study of the influence of anisotropy on the inertial scales of turbulence, J. Fluid Mech. 692, 464 (2012); G. Bellani and E. A. Variano, Homogeneity and isotropy in a laboratory turbulent flow, Exp. Fluids 55, 1646 (2014) and references therein; T. Jamin, Interactions between free-surface waves and hydrodynamics flows, PhD thesis, University of Paris Diderot, 2016; T. Jamin, M. Berhanu, and E. Falcon, Experimental study of hydrodynamic turbulence under a free surface, In preparation.

[15] E. A. Variano and E. A. Cowen, A random-jet-stirred turbulence tank, J. Fluid Mech. 604, 1 (2008).

[16] B. A. Johnson and E. A. Cowen, Turbulent boundary layers absent mean shear, J. Fluid Mech. 835, 217 (2018).

[17] V. Eswaran and S. B. Pope, An examination of forcing in direct numerical simulations of turbulence, Comput. Fluids 16, 257 (1988); K. Alvelius, Random forcing of three-dimensional homogeneous turbulence, Phys. Fluids 11, 1880 (1999); T. Ishihara, T. Gotoh, and Y. Kaneda, Study of high-Reynolds number isotropic turbulence by direct numerical simulation, Annu. Rev. Fluid Mech. 41, 165 (2009); P. K. Yeung, X. M. Zhai, and K. R. Sreenivasan, Extreme events in computational turbulence, Proc. Natl. Acad. Sci. USA 112, 12633 (2015).

[18] T. Lundgren, Linearly forced isotropic turbulence, in Annual Research Brief (Center for Turbulence Research, Standford, 2003), pp 461-473; C. Rosales and C. Meneveau, Linear forcing in numerical simulations of isotropic turbulence: Physical space implementations and convergence properties, Phys. Fluids 17, 095106 (2005); J. Janin, F. Duval, C. Friess, and P. Sagaut, A new linear forcing method for isotropic turbulence with controlled integral length scale, Phys. Fluids 33, 045127 (2021).

[19] Oscillations Modulating Power Law Exponents in Isotropic Turbulence: Comparison of Experiments with Simulations, K. P. Iyer, G. P. Bewley, L. Biferale, K. R. Sreenivasan, and P. K. Yeung, Phys. Rev. Lett. 126, 254501 (2021).

[20] E. Falcon, J.C. Bacri, and C. Laroche, Dissipated power within a turbulent flow forced homogeneously by magnetic particles, Phys. Rev. Fluids 2, 102601(R) (2017).

[21] E. Falcon, J.-C. Bacri, and C. Laroche, Equation of state of a granular gas homogeneously driven by particle rotations, Europhys. Lett. (EPL) 103, 64004 (2013); AIP Conf. Proc. 1542, 815 (2013).

[22] G. Kokot, S. Das, R. G. Winkler, G. Gompper, I. S. Aranson, and A. Snezhko, Active turbulence in a gas of self-assembled spinners, Proc. Natl. Acad. Sci. USA 114, 12870 (2017).

[23] M. Bourgoin, R. Kervil, C. Cottin-Bizonne, F. Raynal, R. Volk, and C. Ybert, Kolmogorovian active turbulence of a sparse assembly of interacting marangoni surfers, Phys. Rev. X 10, 021065 (2020).

[24] S. G. Saddoughi and S. V. Veeravalli, Local isotropy in turbulent boundary layers at high Reynolds number, J. Fluid Mech. 268, 372 (1994); K. R. Sreenivasan, On the universality of the Kolmogorov constant, Phys. Fluids 7, 2778 (1995).

[25] A. N. Kolmogorov, A refinement of previous hypotheses concerning the local structure of turbulence in a viscous incompressible fluid at high Reynolds number, J. Fluid Mech. 13, 82 (1962); A. M. Oboukhov, Some specific features of atmospheric turbulence, J. Fluid Mech. 13, 77 (1962); B. Dubrulle, Intermittency in fully developed turbulence: Logpoisson statistics and generalized scale covariance, Phys. Rev. Lett. 73, 959 (1994); Z.-S. She and E. Lévêque, Universal scaling laws in fully developed turbulence, Phys. Rev. Lett. 72, 336 (1994).

[26] T. von Kármán, and L. Howarth, On the Statistical Theory of Isotropic Turbulence, Proc. R. Soc. Lond. A 164, 192 (1938); R. A. Antonia, and P. Burattini, J. Fluid Mech. 550, 175 (2006); Approach to the 4/5 law in homogeneous isotropic turbulence, M. Sinhuber, G. P. Bewley, and E. Bodenschatz, Dissipative Effects on Inertial-Range Statistics at High Reynolds Number, Phys. Rev. Lett. 119, 134502 (2017).

[27] M. Raffel, C. E. Willert, S. T. Wereley, and J. Kompenhans, Particle Image Velocimetry: A practical guide, 2nd Ed. (Springer-Verlag, Berlin, 2007).

[28] See Supplemental Material at http://link.aps.org/... for movies and further data analyses.

[29] H. Tennekes, Eulerian and lagrangian time microscales in isotropic turbulence, J. Fluid Mech. 67, 561 (1975).

[30] J. C. H. Fung, J. C. R. Hunt, N. A. Malik, and R. J. Perkins, Kinetic simulation of homogeneous turbulence by unsteady random Fourier modes, J. Fluid Mech. 236, 281 (1992).

[31] K. P. Iyer, K. R. Sreenivasan, and P. K. Yeung, Refined similarity hypothesis using three-dimensional local averages, Phys. Rev. E 92, 063024 (2015).

[32] K. P. Iyer, K. R. Sreenivasan, and P. K. Yeung, Scaling exponents saturate in three-dimensional isotropic turbulence, Phys. Rev. Fluids 5, 054605 (2020).

[33] P. L. O’Neill, D. Nicolaides, D. R. Honnery, and J. Soria, Autocorrelation functions and the determination of integral length with reference to experimental and numerical data, In Proc. 15th Australasian Fluid Mech. Conf., edited by M. Behnia, W. Lin, and G. D. McBain (University of Sydney, Australia, 2004) pp. 1-4.

[34] G. I. Taylor, Statistical theory of turbulence, Proc. R. Soc. A 151, 421 (1935).

[35] B. R. Pearson, P.-A. Krogstad, and W. van de Water, Measurements of the turbulent energy dissipation rate, Phys. Fluids 14, 1288 (2002)

[36] K. R. Sreenivasan, On the scaling of the turbulence energy dissipation rate, Phys. Fluids 27, 1048 (1984).

[37] D. Lohse, Crossover from high to low reynolds number turbulence, Phys. Rev. Lett. 73, 3223 (1994).

[38] G. Wang, F. Yang, K. Wu, Y. Ma, C. Peng, T. Liu, and L.-P. Wang, Estimation of the dissipation rate of turbulent kinetic energy: A review, Chem. Eng. Sci. 229, 116133 (2021). 
[39] M. M. Hoque, M. J. Sathe, S. Mitra, J. B. Joshi, and G. M. Evans, Comparison of specific energy dissipation rate calculation methodologies utilising 2D PIV velocity measurement, Chem. Eng. Sci. 137, 752 (2015).

[40] G. K. Batchelor, A. A. Townsend, and H. Jeffreys, The nature of turbulent motion at large wave-numbers, Proc. R. Soc. Lond. A 199, 238 (1949); A. Arnéodo, R. Benzi, J. Berg, L. Biferale, E. Bodenschatz, A. Busse, and et al. (International Collaboration for Turbulence Research), Universal intermittent properties of particle trajectories in highly turbulent flows, Phys. Rev. Lett. 100, 254504 (2008).

[41] F. Toschi and E. Bodenschatz, Lagrangian properties of particles in turbulence, Annu. Rev. Fluid Mech. 41, 375 (2009).

[42] V. Dallas, S. Fauve, and A. Alexakis, Statistical equilibria of large scales in dissipative hydrodynamic turbulence, Phys. Rev. Lett. 115, 204501 (2015); A. Alexakis and M.-E. Brachet, On the thermal equilibrium state of large-scale flows, J. Fluid Mech. 872, 594 (2019); Energy fluxes in quasi-equilibrium flows. J. Fluid Mech. 884, A33 (2020).

[43] M. Buzzicotti, L. Biferale, and F. Toschi, Statistical properties of turbulence in the presence of a smart small-scale control, Phys. Rev. Lett. 124, 084504 (2020). 


\title{
SUPPLEMENTAL MATERIAL \\ "Three-dimensional turbulence generated homogeneously by magnetic particles"
}

\author{
A. Cazaubiel, J.-B. Gorce, J.-C. Bacri, M. Berhanu, C. Laroche, and E. Falcon \\ Université de Paris, MSC, UMR 7057 CNRS, F-75 013 Paris, France
}

In this supplemental material, we present movies of fluid tracer trajectories (Sec. I). Additional characteristics on mean flow and isotropy are also displayed (Sec. III), followed by details on the scaling of the turbulence level with the forcing parameters (Sec. III) and the scaling of the energy spectrum with the forcing parameters (Sec. IV).

\section{MOVIES}

Movies of fluid tracer trajectories are shown for an increasing energy input (frequency $F$ and amplitude $B$ of the magnetic field) for $N=10$ magnetic particles during $3.3 \mathrm{~s}$ (slow down 3 times). The fluid flow is visualized using Polyamide fluid tracers $(50 \mu \mathrm{m})$ illuminated by a horizontal laser sheet. A high-resolution video camera (Phantom V10, $2400 \times 1800$ pixels $\left.^{2}-200 \mathrm{fps}\right)$ records the motion of the fluid tracers. Bright dots correspond to the maximal pixel value of tracers averaged over 10 consecutive images $(0.05 \mathrm{~s})$. Window size $=9.4 \times 8.4 \mathrm{~cm}^{2}$. Note the rare events of rotating magnetic particles passing through the laser sheet. The fluid velocity is maximal in the vicinity of the magnetic particles.

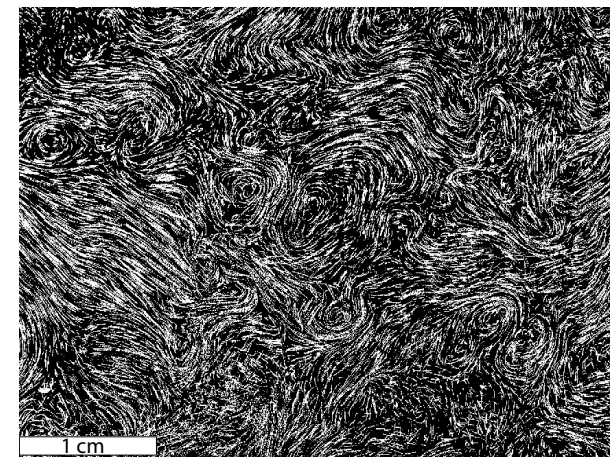

- stack5.96A20HzN10.avi: Low forcing $\sigma_{u}=1.6 \mathrm{~cm} / \mathrm{s}(F=20 \mathrm{~Hz}, B=137 \mathrm{G})$,

- stack4.25A40HzN10.avi: Medium forcing $\sigma_{u}=2.8 \mathrm{~cm} / \mathrm{s}(F=40 \mathrm{~Hz}, B=98 \mathrm{G})$,

- stack7.48A50HzN10.avi: Strong forcing $\sigma_{u}=3.8 \mathrm{~cm} / \mathrm{s}(F=50 \mathrm{~Hz}, B=172 \mathrm{G})$.

Side and top views of an encapsulated magnetic particle $(1 \mathrm{~cm})$ :

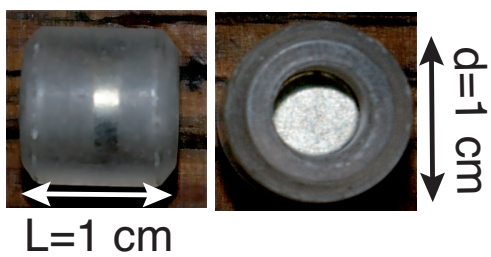




\section{MEAN FLOW AND ISOTROPY}
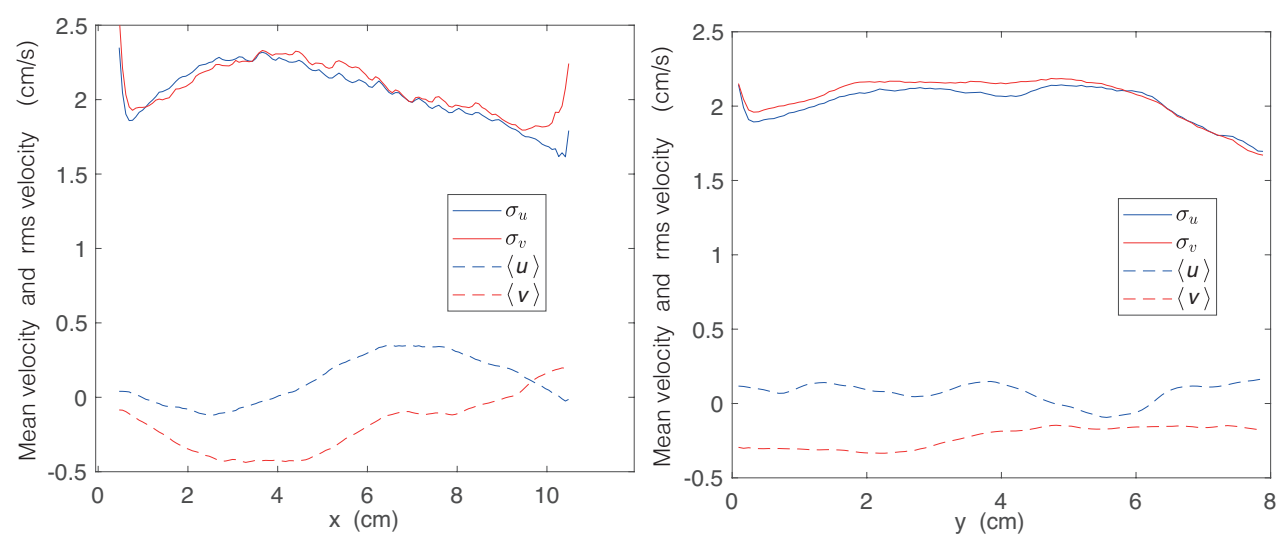

FIG. S1: Mean velocity fields $\left[\langle u\rangle_{t} ;\langle v\rangle_{t}\right]$ and RMS velocity fluctuations $\left(\sigma_{u} ; \sigma_{v}\right)$ of the fluid as a function of the coordinates $(x)$ and $(y)$ in the horizontal plane. $\sigma_{i} \equiv \sqrt{\left\langle i^{2}\right\rangle_{t}-\langle i\rangle_{t}^{2}}$, where $i=u$ or $v$. The value of the velocities are averaged for $13 \mathrm{~s}$. PIV measurements. The mean velocities are found to be much smaller than the RMS fluctuations (i.e., $\langle i\rangle_{t, x} /\left\langle\sigma_{i}\right\rangle_{x}<11 \%$ ) to be able to neglect the mean flow. $\sigma_{u} \approx \sigma_{v}$ is found also to be roughly constant far from the container boundaries located at $x=0$ and $x=11 \mathrm{~cm}$. The isotropy ratios are $\left\langle\sigma_{u} / \sigma_{v}\right\rangle_{x}=0.96$ and $\left\langle\sigma_{u} / \sigma_{v}\right\rangle_{y}=0.98$. The velocity field is thus isotropic in the horizontal plane, the domains close to the boundaries being excluded in the computations.

\section{TURBULENCE LEVEL WITH THE FORCING PARAMETERS}
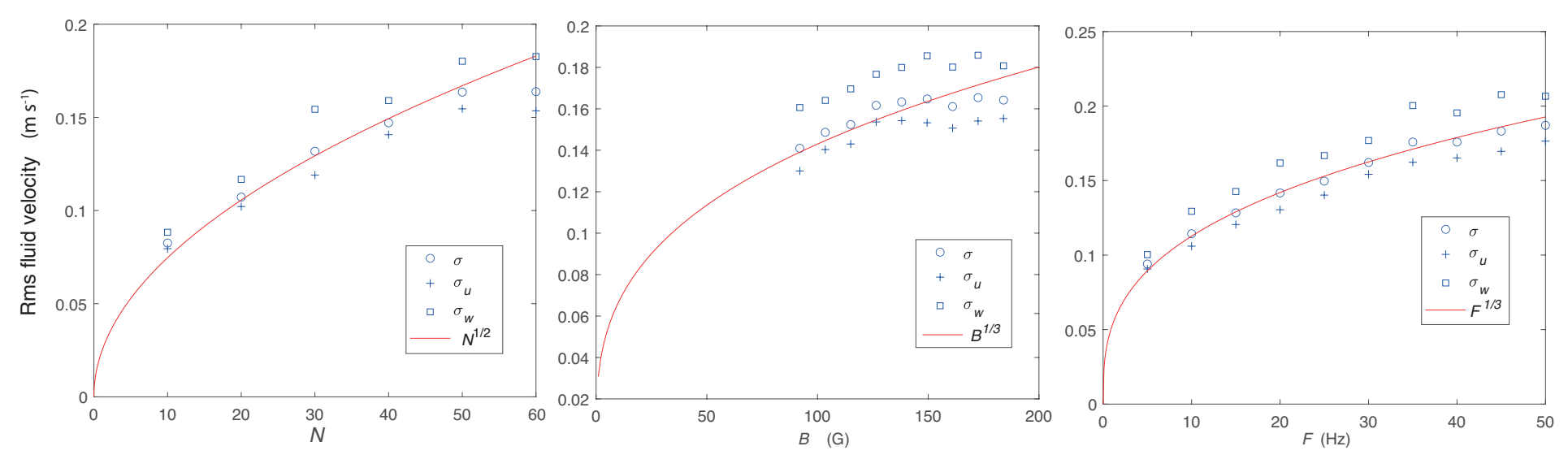

FIG. S2: Scaling of the RMS fluid velocity fluctuations as a function of the forcing parameters. Longitudinal $\sigma_{u}$, vertical $\sigma_{w}$, and total, $\sigma \equiv \sqrt{\left(\sigma_{u}^{2}+\sigma_{v}^{2}+\sigma_{w}^{2}\right) / 3}$, RMS velocity fluctuations as a function of (a) the number $N$ of magnetic particles (for fixed $B=161 \mathrm{G}, F=30 \mathrm{~Hz}$ ), (b) the magnetic field strength $B$ (for fixed $N=60, F=30$ $\mathrm{Hz}$ ), and (c) the magnetic field frequency $F$ (for fixed $N=60, B=161 \mathrm{G}$ ). Solid lines display the best fits leading to $\sigma \sim N^{1 / 2} B^{1 / 3} F^{1 / 3}$. The transverse velocity coordinate is not shown since $\sigma_{v} \approx \sigma_{u}$ (see Fig. S1). LDV measurements were performed at the center of the horizontal plane and a distance of $3.5 \mathrm{~cm}$ above the bottom of the container. 


\section{ENERGY SPECTRUM SCALING WITH THE FORCING PARAMETERS}
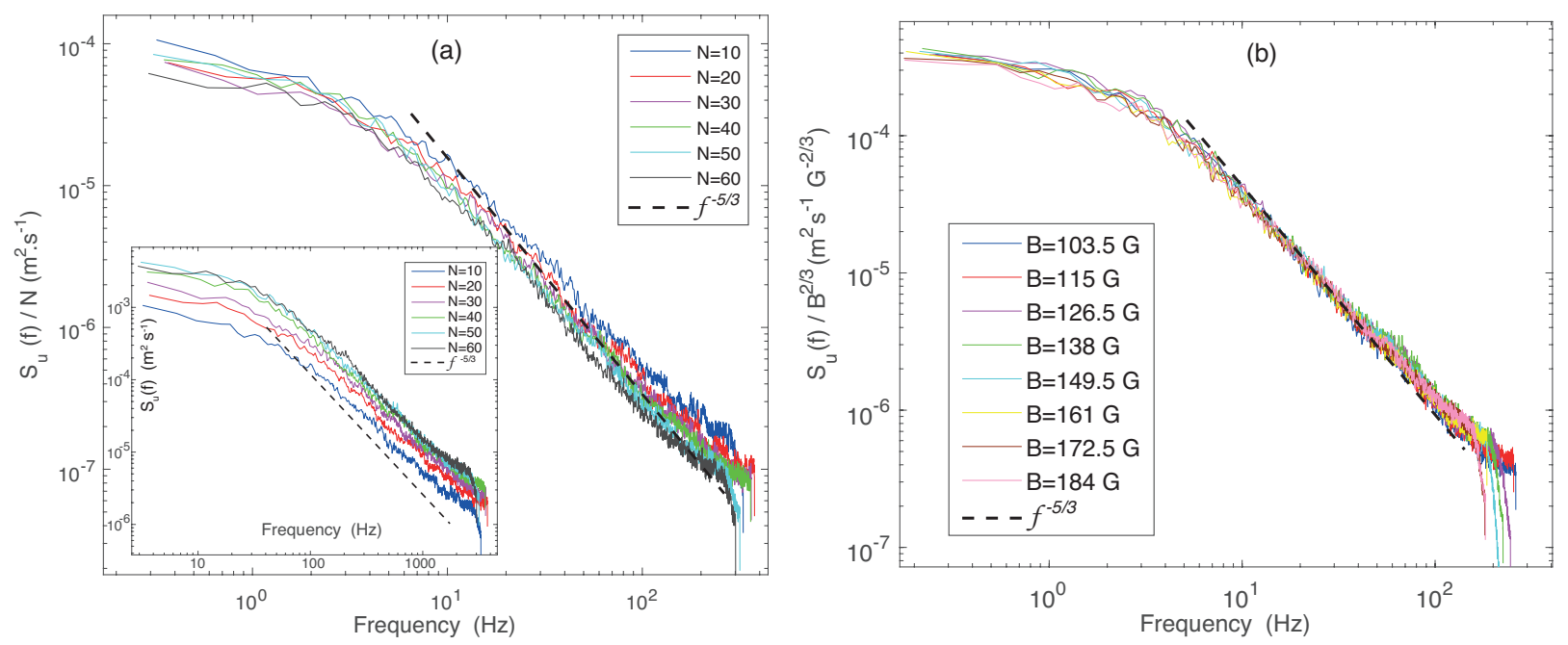

FIG. S3: (a) Frequency power spectrum density $S_{u}(f)$ rescaled by $N$ for different magnetic particles $N \in[10,60]$ (for fixed $B=161 \mathrm{G}, F=30 \mathrm{~Hz}$ ). Inset: Same unrescaled. (b) $S_{u}(f)$ rescaled by $B^{2 / 3}$ for different magnetic field strength $B \in[103,184] \mathrm{G}$ (for fixed $N=60, F=30 \mathrm{~Hz}$ ). The spectra $S_{u}(f)$ are well superimposed when rescaled by (a) $N$ and (b) $B^{2 / 3}$ as expected from $\sigma \equiv\left[\int S_{u}(f, t) d f\right]^{1 / 2}$ with $\sigma \sim N^{1 / 2} B^{1 / 3} F^{1 / 3}$ (see Fig. S2 $)$. See Fig. 3 of the main article for the full rescaled and compensated spectra. LDV measurements. Dashed lines correspond to a $f^{-5 / 3}$ scaling from the Kolmogorov's spectrum [3] and the Tennekes' model [29] (see text of the main article). 